\title{
Plasma, Urine, and Adipose Tissue Biomarkers of Dietary Intake Differ Between Vegetarian and Non-Vegetarian Diet Groups in the Adventist Health Study-2
}

\author{
Fayth L Miles, 1,2,3 Jan Irene C Lloren, ${ }^{1,2}$ Ella Haddad,,2 Karen Jaceldo-Siegl, ${ }^{1,2}$ Synnove Knutsen,,2 \\ Joan Sabate, ${ }^{1,2}$ and Gary E Fraser ${ }^{1,2,4}$ \\ ${ }^{1}$ Adventist Health Study, School of Public Health; ${ }^{2}$ Center for Nutrition, Healthy Lifestyle, and Disease Prevention, School of Public \\ Health; ${ }^{3}$ Department of Preventive Medicine, School of Medicine; and ${ }^{4}$ Department of Medicine, School of Medicine, Loma Linda \\ University, Loma Linda, CA
}

\begin{abstract}
Background: Differences in food composition, nutrient intake, and various health outcomes have been reported for vegetarians and non-vegetarians in the Adventist Health Study-2 (AHS-2) cohort.

Objective: We sought to determine whether biomarkers of dietary intake also differed between individuals classified as vegetarian (vegan, lacto-ovo-vegetarian, pesco-vegetarian, semi-vegetarian) and non-vegetarians based on patterns of consumption of meat, dairy, and eggs.

Methods: Fasting plasma, overnight urine, and adipose tissue samples were collected from a representative subset of AHS-2 participants classified into 5 diet groups (vegan, lacto-ovo-vegetarian, pesco-vegetarian, semi-vegetarian, non-vegetarian) who also completed food-frequency questionnaires. Diet-related biomarkers including carotenoids, isoflavones, enterolactone, saturated and polyunsaturated fatty acids, and vitamins were analyzed in 840 male and female participants. Multiple linear regression was used to examine the association between diet pattern and biomarker abundance, comparing each of 4 vegetarian dietary groups to non-vegetarians, and adjusted mean values were calculated. Bonferroni correction was applied to control for multiple testing.
\end{abstract}

Results: Vegans had higher plasma total carotenoid concentrations (1.6-fold, $P<0.0001$ ), and higher excretion of urinary isoflavones (6-fold, $P<0.0001$ ) and enterolactone (4.4-fold) compared with non-vegetarians. Vegans had lower relative abundance of saturated fatty acids including myristic, pentadecanoic, palmitic, and stearic acids $(P<0.0001)$. Vegans had higher linoleic acid (18:2 $\omega$-6) relative to non-vegetarians $(23.3 \%$ compared with $19.1 \%)(P<0.0001)$, and a higher proportion of total $\omega$-3 fatty acids $(2.1 \%$ compared with $1.6 \%)(P<0.0001)$. Results overall were similar but less robust for lacto-ovo- and pesco-vegetarians. 1-Methylhistidine was $92 \%$ lower in vegans, and lower in lacto-ovo- and pescovegetarians by $90 \%$ and $80 \%$, respectively, relative to non-vegetarians $(P<0.0001)$.

Conclusion: AHS-2 participants following vegan, and lacto-ovo- or pesco-vegetarian diet patterns have significant differences in plasma, urine, and adipose tissue biomarkers associated with dietary intakes compared with those who consume a non-vegetarian diet. These findings provide some validation for the prior classification of dietary groups within the AHS-2 cohort. J Nutr 2019;149:667-675.

Keywords: diet patterns, vegetarians, biomarkers, Adventist Health Study-2 cohort, linear regression,

phytochemicals

\section{Introduction}

Vegetarian diet patterns are characterized not simply by the absence of meat and/or dairy products but also by increased consumption of plant foods. There is growing evidence that such plant-based diet patterns are associated with a number of favorable health effects. Various epidemiological studies conducted with the Adventist Health Study-2 (AHS-2) cohort have demonstrated that vegetarian diet patterns are associated with reduced risk of colorectal and other cancers (1-3), diabetes (4), and metabolic syndrome, reflected by decreased blood pressure, triglycerides, glucose, and waist circumference $(5,6)$.

The favorable effects of vegetarian diets are largely attributable to the increased amount of phytochemicals present in plant foods, besides the increases in fiber and other nutrients. For example, increased consumption of fruits and vegetables 
is positively correlated with carotenoids in blood (7-11) and flavonoids in urine (12), which have anti-inflammatory and antioxidant activity, neutralizing free radicals and protecting against cell damage and chronic diseases. Other polyphenols are elevated in urine or serum with increased consumption of plant foods, including isoflavones, present in soy (13-15), and lignans, found in seeds, whole grains, fruits, vegetables, and nuts (16, 17). These also have protective effects against cardiovascular disease, cancer, and other chronic diseases $(18,19)$. Plant-based diets have also been associated with more favorable profiles of fatty acids $(20,21)$.

Concentrations of dietary biomarkers may be affected by other biological or lifestyle factors (metabolism, hormone signaling, digestive health, the microbiome, etc.) and thus are not directly indicative of nutrient intake, but nonetheless have considerable utility in providing a more accurate assessment of dietary intake. This is particularly relevant in light of the inevitable measurement error associated with FFQs. Beyond the benefit of increasing validity in measurement of dietary intake, the analysis of such biomarkers is highly valuable in clinical and epidemiological studies in 1) validating the classification of individuals by dietary group or pattern, 2) supporting inferences regarding associations between dietary intake and health outcomes, and 3) elucidating the relation between diet and disease pathways by identification of clinically relevant bioactive compounds, metabolites, or precursors.

We previously demonstrated that vegetarians within the AHS-2 cohort, and especially vegans, consumed more plant foods including fruits, vegetables, legumes, soy, nuts and seeds, and had lower consumption of animal products (22). We also reported differences in nutrient intakes comparing diet groups (23). Given the considerable differences in food consumption between vegetarians and non-vegetarians, it is reasonable to hypothesize that these dietary groups also have distinct profiles of biomarkers of dietary intake. With the use of plasma, urine, and adipose tissue samples from 840 participants in the AHS-2 cohort classified into 5 diet groups (vegan, lacto-ovo-vegetarian, pesco-vegetarian, semi-vegetarian, non-vegetarian), we sought to determine whether biomarkers of dietary intake differed between these groups.

\section{Methods}

\section{Study design}

Individuals in the current study participated in a calibration study nested within the AHS-2 cohort. The AHS-2 cohort was established between 2002 and 2007, and consists of over 96,000 Seventh Day Adventists aged $\geq 29 \mathrm{y}$. It was designed to examine the effects of diet and diet patterns on health outcomes. Roughly $52 \%$ of participants follow a vegetarian diet (including vegans and lacto-ovo-vegetarians) or partially vegetarian diet (including pesco-vegetarians and semi-vegetarians, with the latter consuming meat more often than once a month but less than once per week), and the others are non-vegetarian. Approximately $60 \%$ of participants are female, and approximately $27 \%$ are black, with $>90 \%$ of others identifying as white. Participants completed a

\footnotetext{
Supported by NIH/National Cancer Institute grant U01CA152939 (to GEF). Author disclosures: FLM, JICL, EH, KJ-S, SK, JS, and GEF, no conflicts of interest.

Supplemental Tables 1 and 2 are available from the "Supplementary data" link in the online posting of the article and from the same link in the online table of contents at https://academic.oup.com/jn/.

Address correspondence to FLM (e-mail: fmiles@llu.edu).

Abbreviations used: AHS-2, Adventist Health Study-2; ALA, $\alpha$-linolenic acid;

DHA, docosahexaenoic acid; EPA, eicosapentaenoic acid.
}

self-administered questionnaire collecting information on diet, physical activity, supplement use, medical history, and demographics at baseline (24).

The calibration subgroup was established between 2004 and 2006 as a representative sample of the parent cohort, but with oversampling of black participants (45\%). Fasting blood, adipose tissue, and overnight urine were collected at field clinics held in local churches (25). Participants were selected by church, and then within the church. Additionally, anthropometric data were collected during clinic visits. Over a period of 9-12 mo, participants completed 2 sets of three 24-h diet recalls through unannounced telephone calls, which were separated by a second FFQ. Additional details of the calibration study have been published previously (26). A total of 909 participants provided biosamples and either complete dietary recalls or a second FFQ. Exclusion of participants with missing data on diet $(n=39)$, race $(n=2)$, education $(n=17)$, age $(n=2)$, caloric intake $(n=21)$, or smoking $(n=12)$ or drinking status $(n=35)$ resulted in an analytic cohort of 840 men and women.

Participants were classified a priori into vegetarian diet groups based on their responses to the FFQ as follows: vegans never or rarely (less than once per month) consumed eggs, dairy, fish, and other meats; lactoovo-vegetarians consumed eggs and dairy more than once per month but fish and other meats less than once per month; pesco-vegetarians consumed fish at least once per month but all other meats less than once a month; semi-vegetarians ate non-fish meats at least once per month and any meat including fish less than once per week; nonvegetarians consumed non-fish meats at least once a month and any meat (including fish) more than once per week. Nutrient intake data were collected with the use of the Nutrition Data System for Research software versions 4.06 and 5.03 (The Nutrition Coordinating Center, University of Minnesota). The study protocol was approved by the institutional review board of Loma Linda University. All participants provided written informed consent.

\section{Biomarkers and laboratory analyses}

Details of laboratory methods for the analysis of biomarker abundance or concentrations have been described previously (26).

We selected plasma, urine, and adipose biomarkers that are commonly associated with consumption of plant-based foods or animal sources. This included carotenoids which are present in fruits and vegetables, isoflavonoids in soy products and legumes, and lignans, found in various plant sources, particularly seeds, whole grains, fruits and vegetables $(7,14,16)$. 1-Methylhistidine is a marker of meat intake (27), particularly red meat and poultry. In addition, we considered saturated fatty acids, which are commonly associated with consumption of animal products or other fatty processed foods, and unsaturated fatty acids, which in contrast are more commonly found in plant sources, such as nuts, seeds, plant-based oils. Furthermore, we analyzed plasma concentrations of vitamins important for overall health which may be obtained from plant-based and/or animal products; holotranscobalamin, and retinol obtained primarily from animal sources (although provitamin A which is present in carotenes can also be converted to retinol), tocopherols which are high in vegetable oils, nuts and seeds, and folic acid enriched in green vegetables, legumes, and citrus fruit (28).

Assays for plasma carotenoids and fatty acids, retinol, and $\alpha$ - and $\gamma$-tocopherols were performed at the University of California, Los Angeles Center for Human Nutrition Biomarker Laboratory. Plasma carotenoids, retinol, and tocopherols were analyzed with HPLC. The intra-assay $\mathrm{CV}$ for the pooled plasma sample was $7.4 \%$ for lutein, $8.7 \%$ for retinol, $8.8 \%$ for $\alpha$-tocopherol, $10.3 \%$ for $\beta$-carotene, $10.6 \%$ for $\gamma$-tocopherol, $11.5 \%$ for $\beta$-cryptoxanthin, $12.2 \%$ for lycopene, and $14.2 \%$ for $\alpha$-carotene (29). Erythrocyte folate was measured with the Immulite Chemiluminescence Immunoassay Analyzer (Siemens Healthcare Diagnostics) as described previously with intra-assay CV of $5.2 \%$ for higher concentrations $(13 \mathrm{ng} / \mathrm{mL})$ and $9.0 \%$ for lower concentrations $(2.1 \mathrm{ng} / \mathrm{mL})$. The detection limits were: $0.05 \mu \mathrm{mol} / \mathrm{L}$ for lutein, $\beta$-cryptoxanthin, lycopene, $\alpha$-carotene, and $\beta$-carotene; $0.3 \mu \mathrm{mol} / \mathrm{L}$ for retinol; $0.7 \mu \mathrm{mol} / \mathrm{L}$ for $\alpha$-tocopherol and $\gamma$-tocopherol; and $0.5 \mathrm{ng} / \mathrm{mL}$ for folate. Total adipose fatty acids were extracted 
with hexane (30) and the resulting methyl esters separated and quantified with use of gas chromatography. The following CV have been established for saturated and polyunsaturated fatty acids: 12:0, $13 \% ; 14: 0,10.5 \% ; 15: 0,12 \% ; 16: 0,1.6 \% ; 16: 1 \omega-7,2.7 \% ; 18: 0$, $2.6 \% ; 18: 1 \omega-9,3.4 \% ; 18: 2 \omega-6,0.7 \% ; 18: 3 \omega-3,0.7 \% ; 20: 4 \omega-6,0.7 \%$; $20: 5 \omega-3,3.2 \% ; 22: 5 \omega-3,4.2 \% ; 22: 6 \omega-3,2.2 \%$. The detection limit was $0.1 \mu \mathrm{mol} / \mathrm{L}$.

Urinary 1-methylhistidine was measured at University of Southern California with a modification of a previously described method for liquid chromatography linked tandem MS (31), with CV $<5 \%$. The detection limit was $0.1 \mu \mathrm{mol} / \mathrm{L}$. Serum holotranscobalamin was measured with an enzyme immunoassay kit (Axis Shield Diagnostics Limited) at the Department of Nutrition Laboratory, Loma Linda University, with intra-assay CV of $7.2 \%$ and inter-assay CV, $10 \%$, and detection limit of $3 \mathrm{pmol} / \mathrm{L}$. Urinary isoflavonoids (daidzein, genistein, equol) and enterolactone were measured at the University of Hawaii by HPLC with photodiode array and mass spectrometry, and adjusted for urinary creatinine concentrations (32). Inter-assay coefficients of variation of $1 \%, 11 \%$, and $13 \%$ were obtained for daidzein, genistein, and equol, respectively, and $8 \%$ for enterolactone (32). Detection limits for isoflavones were $10 \mathrm{nmol} / \mathrm{L}$ or $2-50 \mathrm{pg} / \mathrm{mg}$ creatinine.

\section{Statistical analysis}

Linear regression models were fit for each dietary biomarker of interest to estimate its association with diet pattern, while adjusting for sex, age, race, education, smoking, alcohol drinking, and energy intake (estimated from 24-h diet recalls) to obtain least squares means (adjusted for other covariates in the model) and/or $\beta$ coefficients with $95 \%$ CI. The null hypothesis was that there was no difference in biomarker concentration between any 1 of the 4 vegetarian diets and the non-vegetarian diet, with statistical significance indicated at $\alpha<0.05$. A Bonferroni correction was applied to control for multiple comparisons of diet patterns. BMI was also considered as a covariate but not included in the final model as it was found to have inconsequential effects on results. Zero values for biomarker measurements were replaced with a value equivalent to the product of $1 \times 10^{-3}$ and the respective mean value for each biomarker. Biomarkers were $\log$ transformed to improve normality. Because of previously observed differences in biomarker concentrations comparing black and non-black participants (26), and possible differences according to BMI, the effect of race (black compared with non-black) or BMI $\left(<25 \mathrm{~kg} / \mathrm{m}^{2}\right.$ compared with $\geq 25 \mathrm{~kg} / \mathrm{m}^{2}$ ) on the diet pattern-biomarker associations was examined. Heterogeneity tests were conducted by including the respective diet-race or diet-BMI interaction term in the regression model.

All carotenoids (lutein, zeaxanthin, cryptoxanthin, $\alpha$-carotene, $\beta$-carotene, lycopene, and total carotenoids) as well as $\alpha$ - and $\gamma$-tocopherol, were adjusted for serum cholesterol with a residual method to provide values that were uncorrelated with cholesterol. Then, the new response variable for each biomarker $(\mathrm{X})$ was set equal to the sum of the residual and the mean of the $\log$-transformed $\mathrm{X}$. This variable was substituted in subsequent multiple linear regression analyses.

Comparisons of demographic and lifestyle characteristics according to diet pattern were conducted with use of ANOVA for continuous variables and chi-square tests for categorical variables.

Analyses were carried out with use of SAS statistical software package release 9.4 (SAS Institute Inc.).

\section{Results}

Baseline demographic and lifestyle characteristics of participants in the analytic study are shown in Table 1 . The majority of participants in the current study were female $(65 \%)$, a reflection of the parent cohort. There were no notable differences in the proportion of males and females among diet groups. Black participants constituted $43.4 \%$ of the study population, and non-black participants, $56.6 \%$. Differences in the distribution of black and non-black participants across diet groups were observed $(P<0.0007)$. After non-vegetarian, the majority of black participants were pesco-vegetarian, and the majority of non-black participants were lacto-ovo-vegetarian. A higher proportion of semi- and non-vegetarians were former smokers or alcohol drinkers $(P<0.02$ and $P<0.0001)$. Vegans were older and had lower BMI compared to the other diet groups, and non-vegetarians were younger and had higher BMI $(P<0.0003$

TABLE 1 Demographic and lifestyle characteristics of participants in the Calibration Study according to diet pattern ${ }^{1}$

\begin{tabular}{|c|c|c|c|c|c|c|c|}
\hline & All & Vegan & $\begin{array}{l}\text { Lacto-ovo- } \\
\text { vegetarian }\end{array}$ & Pesco-vegetarian & Semi-vegetarian & Non-vegetarian & $P$ \\
\hline Participants, $n(\%)$ & 840 & $72(8.6)$ & $224(26.7)$ & $104(12.4)$ & $38(4.5)$ & $402(47.9)$ & \\
\hline Females & $546(65.0)$ & $49(68.1)$ & $143(63.8)$ & $64(61.5)$ & $27(71.1)$ & $263(65.4)$ & \\
\hline Males & $294(35)$ & $23(31.9)$ & $81(36.2)$ & $40(38.5)$ & $11(29.0)$ & $139(34.6)$ & \\
\hline Ethnicity, $n(\%)$ & & & & & & & 0.0007 \\
\hline Education, $n(\%)$ & & & & & & & 0.47 \\
\hline High school and below & $171(20.4)$ & $13(18.1)$ & $39(17.4)$ & $25(24.0)$ & $10(26.3)$ & $86(20.9)$ & \\
\hline Some college & $340(40.8)$ & $33(45.8)$ & $85(38.0)$ & $36(34.6)$ & $15(39.5)$ & $171(42.5)$ & \\
\hline College graduate and beyond & $329(39.2)$ & $26(36.1)$ & $100(44.6)$ & $43(41.4)$ & $13(34.2)$ & $147(36.6)$ & \\
\hline Smoking, $n(\%)$ & & & & & & & 0.02 \\
\hline Never & $508(60.5)$ & $48(66.7)$ & $168(75.0)$ & $68(65.4)$ & $21(55.3)$ & $203(50.5)$ & \\
\hline Past/former & $278(33.1)$ & $24(33.3)$ & $54(24.1)$ & $30(28.9)$ & $14(36.8)$ & $158(38.8)$ & \\
\hline Current & $54(6.4)$ & $2(0.9)$ & 0 & $6(5.8)$ & $3(7.9)$ & $43(10.7)$ & \\
\hline Age, $y, \pm S D$ & $58.3 \pm 13.3$ & $62.6 \pm 12.0$ & $60.0 \pm 14.1$ & $59.1 \pm 14.0$ & $59.4 \pm 13.5$ & $56.3 \pm 12.5$ & 0.0003 \\
\hline $\mathrm{BMI}, \mathrm{kg} / \mathrm{m}^{2}, \pm \mathrm{SD}$ & $27.4 \pm 6.1$ & $24.7 \pm 4.4$ & $25.7 \pm 5.6$ & $26.5 \pm 5.7$ & $26.8 \pm 4.9$ & $29.2 \pm 6.4$ & 0.0001 \\
\hline Energy, kcal/d, \pm SD & $1596 \pm 500$ & $1694 \pm 418$ & $1703 \pm 516$ & $1512 \pm 546$ & $1561 \pm 467$ & $1545 \pm 485$ & 0.0004 \\
\hline
\end{tabular}

${ }^{1}$ Values are means \pm SDs or $n(\%)$. 
TABLE 2 Biomarkers of dietary intake among participants in the Calibration Study according to diet pattern ${ }^{1,2}$

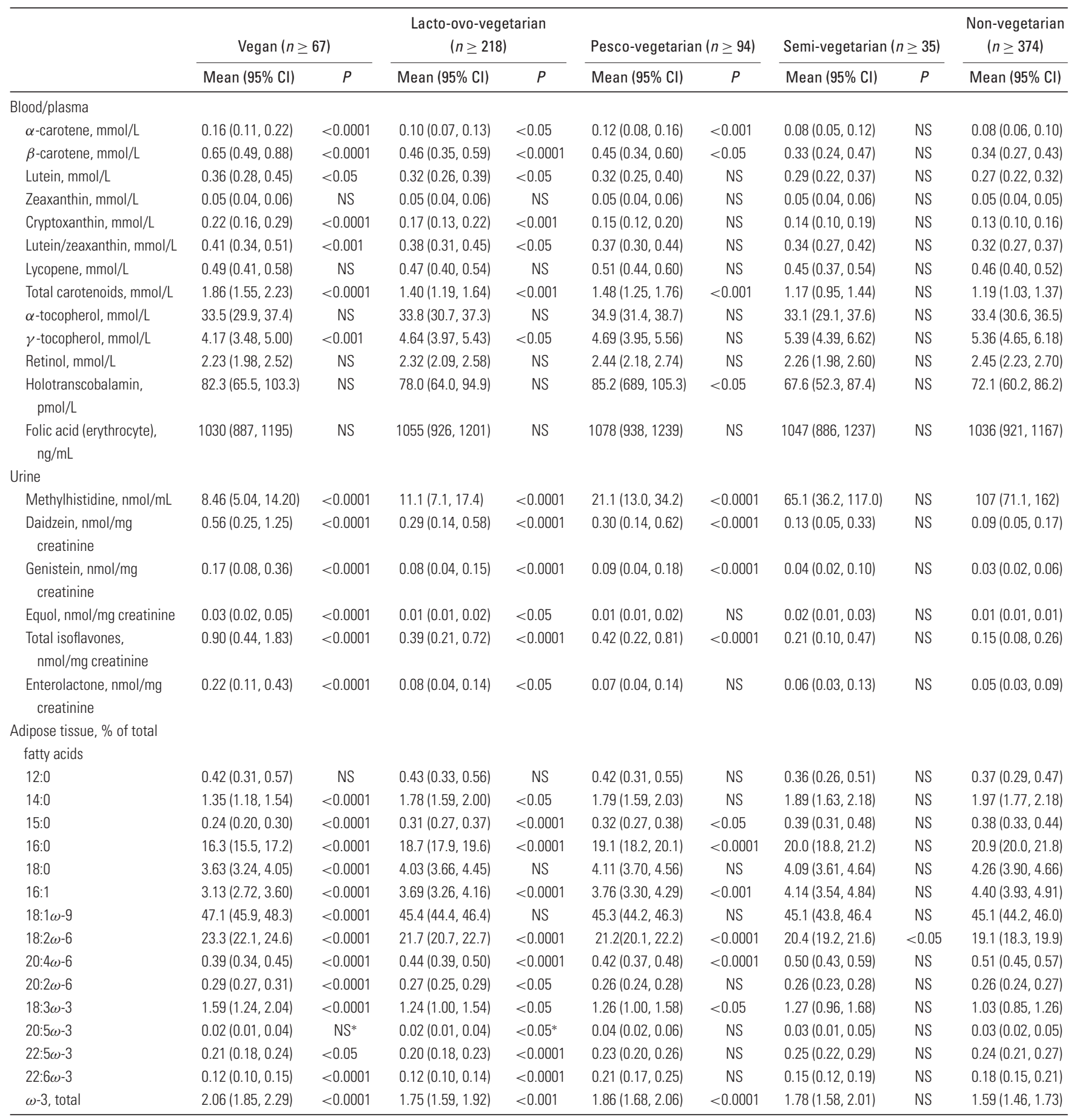

NS, Nonsignificant. 'Values are adjusted geometric means ( $95 \%$ Cls) estimated from linear regression model adjusted for sex, age, race, smoking, alcohol drinking, education, and caloric intake.

${ }^{2} P$ value for difference in adjusted mean value (biomarker) relative to non-vegetarians.

$* P$ value $<0.05$ relative to pesco-vegetarians.

and $P<0.0001)$. Reported energy intake differed among diet groups and was higher in lacto-ovo-vegetarians and vegans $(P<0.0004)$.

A comparison of the adjusted mean values of plasma, urinary, and adipose tissue biomarkers among the 5 diet groups is presented in Table 2. Several differences were noted for plasma carotenoids. Concentrations of carotenoids decreased with increasing consumption of animal products, and were lowest in non-vegetarians and semi-vegetarians. Compared to non-vegetarians, vegans had considerably higher concentrations of plasma $\alpha$-carotene ( 2 -fold, $P<0.0001$ ), $\beta$-carotene $(91 \%$, $P<0.0001)$, cryptoxanthin $(69 \%, P<0.0001)$, lutein $(33 \%$, $P<0.05)$, lutein and zeaxanthin $(28 \%, P<0.001)$, and total carotenoids $(56 \%, P<0.0001)$, but a lower concentration of $\gamma$-tocopherol $(22 \%, P<0.001)$. A similar trend could be seen for lacto-ovo-vegetarians, although concentrations were lower than those for vegans and thus differences compared to nonvegetarians were generally less robust [cryptoxanthin and total 
carotenoids $(31 \%$ and $18 \%$ higher, $P<0.001), \alpha$-carotene (25\% higher), lutein (19\% higher), and lutein/zeaxanthin (19\% higher), $(P<0.05), \beta$-carotene $(35 \%$ higher, $P<0.0001)$ and $\gamma$-tocopherol (13.4\% lower, $P<0.05)$ ]. Pesco-vegetarians had significantly higher concentrations of $\alpha$-carotene $(50 \%$, $P<0.001), \beta$-carotene $(32 \%, P<0.05)$, and total carotenoids $(24 \%, P<0.001)$ than non-vegetarians.

Differences were also found in the excretion of urinary biomarkers comparing diet groups, with the most profound differences in vegans for all biomarkers relative to nonvegetarians $(P<0.0001)$. When compared to non-vegetarians, the concentration of 1-methylhistidine was $92.1 \%$ lower in vegans, $89.6 \%$ lower in lacto-ovo-vegetarians, and $80.3 \%$ lower in pesco-vegetarians $(P$ for all $<0.0001)$. Excretion of soy isoflavones daidzein and genistein, as well as total isoflavones was highest in vegans, followed by pesco- and lacto-ovovegetarians, and all differences compared to non-vegetarians were highly significant $(P<0.0001)$. Enterolactone and equol excretion was significantly higher in vegans (4.4-fold and 3 -fold, respectively; $P<0.0001$ ), and to a lesser degree in lacto-ovovegetarians than non-vegetarians.

The proportion of the saturated fatty acid myristic acid $(14: 0)$ was lower in vegans as well as lacto-ovo-vegetarians compared to non-vegetarians $(1.35 \%$ and $1.78 \%$ compared with $1.97 \% ; P<0.0001$ and $P<0.05$, respectively). Pentadecanoic (15:0) and palmitic (16:0) acids were also lower in vegans, lacto-ovo-vegetarians, and pesco-vegetarians, with the lowest values in vegans and lacto-ovo-vegetarians $(P<0.0001)$. Stearic acid $(18: 0)$ was significantly lower in vegans only $(3.63 \%$ compared with $4.26 \% ; P<0.0001)$ compared to non-vegetarians.

Compared to non-vegetarians, the relative proportion of the MUFA palmitoleic acid (16:1) was lower in vegans $(P<0.0001)$, lacto-ovo-vegetarians $(P<0.0001)$, and pescovegetarians $(P<0.001)$. Oleic acid $(18: 1 \omega-9)$ was higher in vegans alone when compared to non-vegetarians, but the increase was marginal (47.1\% compared with $45.1 \%$; $P<0.0001)$. The polyunsaturated fatty acid (PUFA) linoleic acid $(18: 2 \omega-6)$ was higher in all vegetarian groups including vegans, and lacto-ovo-, pesco-, and semi-vegetarians, than nonvegetarians, with the greatest difference in vegans $(23.3 \%$ compared with $19.1 \% ; P<0.0001)$. Eicosadienoic acid $(20: 2 \omega$ $6)$ was also higher in vegans $(P<0.0001)$ and to a lesser extent in lacto-ovo-vegetarians $(P<0.05)$. Arachidonic acid $(20: 4 \omega$ 6) was significantly lower in vegans, lacto-ovo-vegetarians, and pesco-vegetarians $(P<0.0001)$ compared to non-vegetarians.

Among the $\omega$-3 PUFAs, the proportion of $\alpha$-linolenic acid (ALA; 18:3 $\omega$-3) was higher in vegans, lacto-ovo-vegetarians, and pesco-vegetarians compared to non-vegetarians, with the highest values in vegans $(1.59 \%$ compared with $1.03 \%$; $P<0.0001)$. However, docosapentaenoic acid (22:5 $\omega-3)$, and docosahexaenoic acid (DHA; 22:6 $\omega$-3) were significantly lower in vegans $(P<0.05$ and $P<0.0001$, respectively) and lacto-ovovegetarians $(P<0.0001)$. Eicosapentaenoic acid (EPA; 20:5 $\omega$-3) was lower in lacto-ovo-vegetarians relative to non-vegetarians $(P<0.05)$, and was also lower in vegans and lacto-ovovegetarians than in pesco-vegetarians $(P<0.05)$. Nonetheless, total $\omega-3$ was higher in vegans $(2.06 \%$ compared with $1.59 \%$; $P<0.0001)$, followed by pesco-vegetarians $(1.86 \%$ compared with $1.59 \% ; P<0.0001)$ and lacto-ovo-vegetarians $(1.75 \%$ compared with $1.59 \% ; P<0.001)$, relative to non-vegetarians.

We examined the ability of race to modify the effect of diet on biomarker abundance as an exploratory analysis. Black pesco-vegetarians (relative to non-vegetarians) had higher concentrations of $18: 1 \omega-9$ compared to non-blacks $(\beta=0.05, P$ for interaction $<0.0001)$, and black vegans had lower concentrations of zeaxanthin compared to nonblacks $(\beta=-0.4, P$ for interaction $<0.005)$ (Supplemental Table 1).

Examination of the effect of BMI on the association between diet pattern and biomarker concentrations revealed significant effect modification for 18:0 saturated fatty acid among pescoand semi-vegetarians compared to non-vegetarians $(\beta=0.22$ and 0.28 , respectively; $P$ for interaction $<0.005)$. This indicated significantly higher relative concentrations of 18:0 in overweight pesco- and semi-vegetarian participants. Plasma cryptoxanthin concentrations among vegans $(\beta=-0.49, P$ for interaction $<0.05)$ and lacto-ovo-vegetarians $(\beta=-0.42, P$ for interaction $<0.005$ ) compared to non-vegetarians were relatively lower in overweight participants (Supplemental Table 2).

\section{Discussion}

We have shown marked differences in several biomarkers of dietary intake among individuals classified according to dietary patterns depending on the consumption of animal products. Notable distinctions were seen for plasma, urine, and adipose biomarkers comparing vegans, lacto-ovo-vegetarians, and pesco-vegetarians to non-vegetarians. Vegans had the most marked differences in plasma carotenoids $(\alpha$-carotene, $\beta$-carotene, cryptoxanthin, total carotenoids $)(P<0.0001)$, as well as total isoflavones $(P<0.0001)$, and enterolactone $(P<0.0001)$, which were significantly higher than nonvegetarians. In addition, vegans had lower proportions of all saturated fatty acids except lauric acid $(P<0.0001)$. Similar to vegans although less robust, lacto-ovo-vegetarians and pescovegetarians had higher concentrations of total carotenoids $(P<0.001)$ and total isoflavones $(P<0.0001)$. As expected, all 3 of these vegetarian groups had significantly lower 1methylhistidine $(P<0.0001)$. As for PUFAs, the 3 vegetarian groups had lower 20:4 $\omega-6(P<0.0001)$, but significantly higher 18:2 $\omega-6(P<0.0001)$, and higher total $\omega-3(P<0.001)$.

The current findings provide some validation for our previous studies of AHS-2 participants classified according to vegetarian or non-vegetarian dietary patterns. It is clear that the definitions that were used to assign diet groups divide subjects into categories having distinct biological signatures. Previous reports have noted higher consumption of fruits, vegetables, grains, legumes, soy, nuts and seeds among vegetarians, most notably vegans, but also lacto-ovo- and pesco-vegetarians (22). In addition, dietary intakes of $\beta$-carotene and linoleic acid and fiber were found to be highest in vegans and lacto-ovo or pescovegetarians relative to non-vegetarians, whereas saturated fatty acids were lower (23). Our current findings are consistent with these reports.

Higher concentrations of $\beta$-carotene and other carotenoids in plasma have been associated previously with plant-based diets and consumption of plant foods (33-37). Thus we expected to find higher concentrations of $\beta$-carotene and total carotenoids in vegetarians in our study, relative to nonvegetarians, although vegetarians in some studies have shown lower than expected concentrations of carotenoids $(38,39)$. Vegans had the highest isoflavone excretion, as expected, which is strongly associated with soy consumption and vegetarian diets $(8,13,40-42)$. However, concentrations were not as high as those reported for some Asian populations (15), where urinary excretion of total isoflavones was $\sim 300-700 \mathrm{nmol} / \mathrm{h}$ 
for Chinese and Japanese women, consistent with high soy intakes in Asian diets $(15,43)$. About $30 \%$ of the Western adult population are equol producers $(44,45)$ and thus have the gut bacteria necessary for its production from daidzein, compared to $50-60 \%$ of Asian adults $(46,47)$. More than $57 \%$ of our study participants had equol concentrations above $0.01 \mathrm{nmol} / \mathrm{mg}$ creatinine (not shown). Notably, enterolactone was highest in vegans. This is consistent with findings of higher enterolactone with increased consumption of fiber and plant foods $(16,48)$. Although these phytoestrogens are predominantly ingested from plant foods, very small amounts may be obtained from animal products including milk, eggs, meat, and fish/seafood (3-6 $\mu \mathrm{g} / 100 \mathrm{~g}$ wet weight) (49).

Our findings of lower proportions of saturated fatty acids in vegetarians relative to non-vegetarians were expected because of the lower consumption of saturated fats from meat and animal products among vegetarians. As pentadecanoic acid $(15: 0)$ is a marker for dairy consumption (50-52), it was expected that it would be lowest in vegans, and somewhat higher in lacto-ovovegetarians, which was indeed the case. In addition, arachidonic

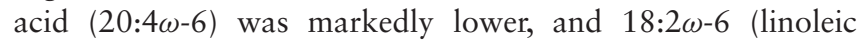
acid) as well as 18:1 (oleic acid) higher among vegans and/or vegetarians. These findings are largely consistent with results from a previous study comparing fatty acid profiles in plasma between Finnish vegans and non-vegetarians, where decreases in saturated fatty acids, and increased $\omega-6$ PUFAs, and MUFAs were observed among vegans (38). There is much evidence that a diet rich in $\omega-6$ PUFA is associated with higher $\omega-6$ PUFA in adipose or plasma lipid fractions (53-58), although greater variability has been observed for saturated fatty acids and MUFAs. Given the correlation with previously reported dietary intakes among AHS-2 participants (26), these biomarkers are likely a reflection of fat intake in the diet. However, fatty acid composition may also be affected by metabolism, genetic variation-such as a polymorphism in an enzyme responsible for endogenous synthesis of PUFAs (59), fat consumption, fatty acid transport and elongation enzymes, gender, BMI, and lifestyle factors such as smoking and alcohol drinking $(60,61)$.

As for the $\omega-3$ fatty acids, higher adipose ALA but lower percentages of DHA, EPA, and their intermediary product, docosapentaenoic acid, were found among vegans and/or lactoovo-vegetarians, also consistent with what has been reported previously for vegans or vegetarians $(38,62)$. The higher percentage of ALA in vegans is not surprising as there are many plant-based sources for ALA, unlike EPA and DHA which are obtained primarily from marine sources, although there are other less common plant sources $(63,64)$. In our study, ALA accounted for the majority of $\omega$-3, explaining why total $\omega$-3 was highest in vegans. Although ALA may be converted to EPA and to a lesser extent DHA, other factors, such as those previously mentioned, may influence conversion efficiency.

It should be noted that many of the biomarkers differing between diet groups showed moderate to strong correlations with dietary intakes based on FFQs and 24-h diet recalls in our previous calibration study (26), further supporting the findings of the current study. We previously reported high correlations between meat intake and urinary 1-methylhistidine, and therefore we expected 1-methylhistidine to be significantly lower in vegetarians, consistent with our current results. Urinary isoflavones which differed significantly between vegetarians and non-vegetarians in the current study also previously showed moderate correlations with 24-h diet recalls, and adipose fatty acids 18:2, 18:3, and 14:0 had moderate to strong correlations with dietary intakes (26).
We often deal with validity issues in dietary assessment studies. Under- or over-reporting is common, attributable to subjects' perceptions of social desirability, poor recall, questionnaire design, and other issues. This is likely a contributor to the lack of coherence between reported energy intakes and BMI, as energy intake is difficult to measure accurately with FFQs and poor correlations have been found when comparing reported energy intake in FFQs with 24-h diet recalls (24). However, the discordance may also be a reflection of the difference in the rate of metabolism on a plant-based compared to non-vegetarian diet, as there is evidence of higher calorie expenditure on a vegan diet even if calorie intake is higher, in the presence of limited fat consumption (65). Twenty four-hour diet recalls, although more accurate, may not reflect diet patterns or vegetarian status dependent on exclusion of low intakes of some foods. Approaches and methods have been developed to improve accuracy of assessment of dietary intake, and we have previously discussed calibration methods to address measurement error issues (66). Although biomarkers are not without shortcomings, they supplement other validation methods and may sometimes help to circumvent the biasing effects of diet recall by providing more accurate measures of intake of selected nutrients (67).

Although biomarkers may be strongly associated with diet patterns, as with the adipose fatty acids, they do not solely reflect the diet, but are also affected by absorption, endogenous metabolism, and the gut microbiome, among other factors. Notwithstanding, biomarkers are very valuable in understanding disease etiology, and may provide a more secure foundation for causal inferences about diet-disease relations in epidemiologic studies. By this measure, the findings reported here are of value, given previously reported associations between vegetarian diet patterns and health outcomes, including apparent reductions of cancer incidence, cardiometabolic risk factors, type 2 diabetes, and overall mortality (68). It is understood that phytochemicals play an important role in prevention of these diseases. There is considerable evidence, particularly for the anticancer, anti-inflammatory, and antioxidant activities of carotenoids, polyphenols, and isoflavones $(69,70)$. In this manner the current study provides some validation of our previous findings of favorable health outcomes in individuals following a plant-based diet by demonstrating selective increases in such bioactive markers that are related to consumption of plant foods.

A major strength of this study is the large sample size with biomarker measurements for over 800 participants, and sufficient power to detect changes between diet pattern groups. Another considerable strength is the detailed dietary assessment of study participants, who completed a baseline FFQ, reflective of longer-term dietary habits, enabling a priori classification of participants by dietary pattern. However, although individuals were assigned vegetarian status a priori based on reported dietary intakes, there is always the possibility of some information bias and consequently misclassification of diet group. Another limitation was the limited power to detect interactions between the biomarker-diet pattern association and race or BMI, in particular because of the lower numbers of vegans, and thus no clear conclusions can be drawn. In addition, urine samples represented 12-h overnight collections, which must be taken into consideration when comparing urinary biomarkers examined in this study with other populations. Plasma and urine samples were obtained at only one timepoint, and consequently biomarker measurements in individuals may not be totally reflective of diet status. Within-person variation was not accounted for. As mentioned above, because biomarker 
concentrations may be affected by many factors, some caution must be exercised with using these data in support of causal inferences about dietary patterns and health outcomes.

Additional considerations include temporal differences associated with metabolism or absorption of these biomarkers. Urine metabolites may be a better reflection of acute dietary intake (relative to plasma and adipose). Elimination half-lives of isoflavones and enterolignans are relatively short (6-8 and 4-13 h, respectively) $(71,72)$. Plasma carotenoid half-lives are longer, ranging from $\leq 12$ to $>60 \mathrm{~d}$. Differences in plasma metabolites are more easily detected with long-term as opposed to acute interventions (73), which is more comparable to the long-term diet patterns examined in the current cohort. Adipose tissue reflects longer-term storage of fats (74). Fatty acids generally have a long half-life and may be incorporated into cell membranes, and represent an integrated measure of up to $2 \mathrm{y}$ of dietary intake, although correlations with dietary intake may vary $(60,61)$.

In conclusion, we report significant and overall favorable differences in several biomarkers associated with nutrient intake, including carotenoids, isoflavones, enterolactone, and various fatty acids in vegetarians, particularly vegans compared to non-vegetarians. Our findings also provide some validation of the dietary patterns represented in the AHS- 2 cohort, and may help to elucidate the significance of diet-related biomarkers in disease prevention.

\section{Acknowledgments}

The authors' responsibilities were as follows-FLM: wrote the manuscript and performed the statistical analysis; GEF: designed and guided the research and analysis; GEF, SK, and JS: designed the initial calibration study; JICL: provided, organized, and processed computational data; EH: managed the biorepository; and all authors: read and approved the final paper.

\section{References}

1. Orlich MJ, Singh PN, Sabate J, Fan J, Sveen L, Bennett H, Knutsen SF, Beeson WL, Jaceldo-Siegl K, Butler TL, et al. Vegetarian dietary patterns and the risk of colorectal cancers. JAMA Intern Med 2015;175(5):76776.

2. Tantamango-Bartley Y, Jaceldo-Siegl K, Fan J, Fraser G. Vegetarian diets and the incidence of cancer in a low-risk population. Cancer Epidemiol Biomarkers Prev 2013;22(2):286-94.

3. Tantamango-Bartley Y, Knutsen SF, Knutsen R, Jacobsen BK, Fan J, Beeson WL, Sabate J, Hadley D, Jaceldo-Siegl K, Penniecook J, et al. Are strict vegetarians protected against prostate cancer? Am J Clin Nutr 2016;103(1):153-60.

4. Tonstad S, Stewart K, Oda K, Batech M, Herring RP, Fraser GE. Vegetarian diets and incidence of diabetes in the Adventist Health Study2. Nutr Metab Cardiovasc Dis 2013;23(4):292-9.

5. Fraser G, Katuli S, Anousheh R, Knutsen S, Herring P, Fan J. Vegetarian diets and cardiovascular risk factors in black members of the Adventist Health Study-2. Public Health Nutr 2015;18(3):537-45.

6. Rizzo NS, Sabate J, Jaceldo-Siegl K, Fraser GE. Vegetarian dietary patterns are associated with a lower risk of metabolic syndrome: the Adventist Health Study-2. Diabetes Care 2011;34(5):1225-7.

7. Broekmans WM, Klopping-Ketelaars IA, Schuurman CR, Verhagen H, van den Berg H, Kok FJ, van Poppel G. Fruits and vegetables increase plasma carotenoids and vitamins and decrease homocysteine in humans. J Nutr 2000;130(6):1578-83.

8. Souverein OW, de Vries JH, Freese R, Watzl B, Bub A, Miller ER, Castenmiller JJ, Pasman WJ, van Het Hof K, Chopra M, et al. Prediction of fruit and vegetable intake from biomarkers using individual participant data of diet-controlled intervention studies. Br J Nutr 2015;113(9):1396-409.
9. Campbell DR, Gross MD, Martini MC, Grandits GA, Slavin JL, Potter JD. Plasma carotenoids as biomarkers of vegetable and fruit intake. Cancer Epidemiol Biomarkers Prev 1994;3(6):493-500.

10. Carlsen MH, Karlsen A, Lillegaard IT, Gran JM, Drevon CA, Blomhoff $\mathrm{R}$, Andersen LF. Relative validity of fruit and vegetable intake estimated from an FFQ, using carotenoid and flavonoid biomarkers and the method of triads. Br J Nutr 2011;105(10):1530-8.

11. Couillard C, Lemieux S, Vohl MC, Couture P, Lamarche B. Carotenoids as biomarkers of fruit and vegetable intake in men and women. Br J Nutr 2016;116(7):1206-15.

12. Nielsen SE, Freese R, Kleemola P, Mutanen M. Flavonoids in human urine as biomarkers for intake of fruits and vegetables. Cancer Epidemiol Biomarkers Prev 2002;11(5):459-66.

13. Morimoto Y, Beckford F, Franke AA, Maskarinec G. Urinary isoflavonoid excretion as a biomarker of dietary soy intake during two randomized soy trials. Asia Pac J Clin Nutr 2014;23(2):205-9.

14. Jaceldo-Siegl K, Fraser GE, Chan J, Franke A, Sabate J. Validation of soy protein estimates from a food-frequency questionnaire with repeated 24-h recalls and isoflavonoid excretion in overnight urine in a Western population with a wide range of soy intakes. Am J Clin Nutr 2008;87(5):1422-7.

15. Maskarinec G, Singh S, Meng L, Franke AA. Dietary soy intake and urinary isoflavone excretion among women from a multiethnic population. Cancer Epidemiol Biomarkers Prev 1998;7(7):613-9.

16. Tetens I, Turrini A, Tapanainen H, Christensen T, Lampe JW, Fagt $S$, Hakansson N, Lundquist A, Hallund J, Valsta LM, et al. Dietary intake and main sources of plant lignans in five European countries. Food Nutr Res 2013;57:19805 - http://dx.doi.org/10.3402/fnr.v57i0.19805.

17. Peterson J, Dwyer J, Adlercreutz H, Scalbert A, Jacques P, McCullough ML. Dietary lignans: physiology and potential for cardiovascular disease risk reduction. Nutr Rev 2010;68(10):571-603.

18. Kaulmann A, Bohn T. Carotenoids, inflammation, and oxidative stress-implications of cellular signaling pathways and relation to chronic disease prevention. Nutr Res 2014;34(11):907-29.

19. Yoder S, Lancaster S, Hullar M, Lampe J. Gut microbial metabolism of plant lignans: influence on human health. Edition ed. In: Rio KTDD , editor. Diet-Microbe Interactions In The Gut Effects on Human Health and Disease. Elsevier, Inc.; 2015, 103-17.

20. Ambring A, Johansson M, Axelsen M, Gan L, Strandvik B, Friberg P. Mediterranean-inspired diet lowers the ratio of serum phospholipid n-6 to n-3 fatty acids, the number of leukocytes and platelets, and vascular endothelial growth factor in healthy subjects. Am J Clin Nutr 2006;83(3):575-81.

21. Harper CR, Jacobson TA. Beyond the Mediterranean diet: the role of omega-3 fatty acids in the prevention of coronary heart disease. Prev Cardiol 2003;6(3):136-46.

22. Orlich MJ, Jaceldo-Siegl K, Sabate J, Fan J, Singh PN, Fraser GE. Patterns of food consumption among vegetarians and non-vegetarians. Br J Nutr 2014;112(10):1644-53.

23. Rizzo NS, Jaceldo-Siegl K, Sabate J, Fraser GE. Nutrient profiles of vegetarian and nonvegetarian dietary patterns. J Acad Nutr Diet 2013;113(12):1610-9.

24. Jaceldo-Siegl K, Knutsen SF, Sabate J, Beeson WL, Chan J, Herring RP, Butler TL, Haddad E, Bennett H, Montgomery S, et al. Validation of nutrient intake using an FFQ and repeated $24 \mathrm{~h}$ recalls in black and white subjects of the Adventist Health Study-2 (AHS-2). Public Health Nutr 2010;13(6):812-9.

25. Chan J, Knutsen SF, Sabate J, Haddad E, Yan R, Fraser GE. Feasibility of running clinics to collect biological specimens in a nationwide cohort study-Adventist Health Study-2. Ann Epidemiol 2007;17(6):454-7.

26. Fraser GE, Jaceldo-Siegl K, Henning SM, Fan J, Knutsen SF, Haddad $\mathrm{EH}$, Sabate J, Beeson WL, Bennett H. Biomarkers of dietary intake are correlated with corresponding measures from Repeated Dietary Recalls and Food-Frequency Questionnaires in the Adventist Health Study-2. J Nutr 2016;146(3):586-94.

27. Myint T, Fraser GE, Lindsted KD, Knutsen SF, Hubbard RW, Bennett HW. Urinary 1-methylhistidine is a marker of meat consumption in black and in white California Seventh-day Adventists. Am J Epidemiol 2000;152(8):752-5.

28. Medical Encyclopedia:Medline Plus. U.S. National Library of Medicine, 2018: https://medlineplus.gov/encyclopedia.html.

29. Lu QY, Hung JC, Heber D, Go VL, Reuter VE, Cordon-Cardo C, Scher HI, Marshall JR, Zhang ZF. Inverse associations between plasma 
lycopene and other carotenoids and prostate cancer. Cancer Epidemiol Biomarkers Prev 2001;10(7):749-56.

30. Bagga D, Capone S, Wang HJ, Heber D, Lill M, Chap L, Glaspy JA. Dietary modulation of omega-3/omega- 6 polyunsaturated fatty acid ratios in patients with breast cancer. J Natl Cancer Inst 1997;89(15):1123-31.

31. Suh J, J Walsh W, R McGinnis W, Allen L, Ames B. Altered sulfur amino acid metabolism in immune cells of children diagnosed with autism. Am J Biochem Biotechnol 2008;4(2):105-13.

32. Franke AA, Custer LJ, Wilkens LR, Le Marchand LL, Nomura AM, Goodman MT, Kolonel LN. Liquid chromatographic-photodiode array mass spectrometric analysis of dietary phytoestrogens from human urine and blood. J Chromatogr B Analyt Technol Biomed Life Sci 2002;777(1-2):45-59.

33. Bach-Faig A, Geleva D, Carrasco J, Ribas-Barba L, Serra-Majem L. Evaluating associations between Mediterranean diet adherence indexes and biomarkers of diet and disease. Public Health Nutr 2006;9(8A):1110-7.

34. Markussen MS, Veierod MB, Sakhi AK, Ellingjord-Dale M, Blomhoff R, Ursin G, Andersen LF. Evaluation of dietary patterns among Norwegian postmenopausal women using plasma carotenoids as biomarkers. Br J Nutr 2015;113(4):672-82.

35. Martini MC, Campbell DR, Gross MD, Grandits GA, Potter JD, Slavin JL. Plasma carotenoids as biomarkers of vegetable intake: the University of Minnesota Cancer Prevention Research Unit Feeding Studies. Cancer Epidemiol Biomarkers Prev 1995;4(5): 491-6.

36. Pierce JP, Natarajan L, Sun S, Al-Delaimy W, Flatt SW, Kealey S, Rock CL, Thomson CA, Newman VA, Ritenbaugh C, et al. Increases in plasma carotenoid concentrations in response to a major dietary change in the women's healthy eating and living study. Cancer Epidemiol Biomarkers Prev 2006;15(10):1886-92.

37. Johnson EJ, Suter PM, Sahyoun N, Ribaya-Mercado JD, Russell RM. Relation between beta-carotene intake and plasma and adipose tissue concentrations of carotenoids and retinoids. Am J Clin Nutr 1995;62(3):598-603.

38. Elorinne AL, Alfthan G, Erlund I, Kivimaki H, Paju A, Salminen I, Turpeinen U, Voutilainen S, Laakso J. Food and nutrient intake and nutritional status of Finnish vegans and non-vegetarians. PLoS One 2016;11(2):e0148235.

39. Szeto YT, Kwok TC, Benzie IF. Effects of a long-term vegetarian diet on biomarkers of antioxidant status and cardiovascular disease risk. Nutrition 2004;20(10):863-6.

40. Adlercreutz H, Fotsis T, Bannwart C, Wahala K, Makela T, Brunow G, Hase T. Determination of urinary lignans and phytoestrogen metabolites, potential antiestrogens and anticarcinogens, in urine of women on various habitual diets. J Steroid Biochem 1986;25(5B):7917.

41. Adlercreutz H, Fotsis T, Watanabe S, Lampe J, Wahala K, Makela T, Hase T. Determination of lignans and isoflavonoids in plasma by isotope dilution gas chromatography-mass spectrometry. Cancer Detect Prev 1994;18(4):259-71.

42. Adlercreutz H, Honjo H, Higashi A, Fotsis $\mathrm{T}$, Hamalainen E, Hasegawa T, Okada H. Urinary excretion of lignans and isoflavonoid phytoestrogens in Japanese men and women consuming a traditional Japanese diet. Am J Clin Nutr 1991;54(6):1093-100.

43. Messina M, Nagata C, Wu AH. Estimated Asian adult soy protein and isoflavone intakes. Nutr Cancer 2006;55(1):1-12.

44. Lampe JW, Karr SC, Hutchins AM, Slavin JL. Urinary equol excretion with a soy challenge: influence of habitual diet. Proc Soc Exp Biol Med 1998;217(3):335-9.

45. Rowland IR, Wiseman H, Sanders TA, Adlercreutz H, Bowey EA. Interindividual variation in metabolism of soy isoflavones and lignans: influence of habitual diet on equol production by the gut microflora. Nutr Cancer 2000;36(1):27-32.

46. Arai Y, Uehara M, Sato Y, Kimira M, Eboshida A, Adlercreutz $\mathrm{H}$, Watanabe S. Comparison of isoflavones among dietary intake, plasma concentration and urinary excretion for accurate estimation of phytoestrogen intake. J Epidemiol 2000;10(2): $127-35$.

47. Song KB, Atkinson C, Frankenfeld CL, Jokela T, Wahala K, Thomas WK, Lampe JW. Prevalence of daidzein-metabolizing phenotypes differs between Caucasian and Korean American women and girls. J Nutr 2006;136(5):1347-51.
48. Miles FL, Navarro SL, Schwarz Y, Gu H, Djukovic D, Randolph TW, Shojaie A, Kratz M, Hullar MAJ, Lampe PD, et al. Plasma metabolite abundances are associated with urinary enterolactone excretion in healthy participants on controlled diets. Food Funct 2017;8(9):3209_ 18.

49. Kuhnle GG, Dell'Aquila C, Aspinall SM, Runswick SA, Mulligan AA, Bingham SA. Phytoestrogen content of foods of animal origin: dairy products, eggs, meat, fish, and seafood. J Agric Food Chem 2008;56(21):10099-104.

50. Brevik A, Veierod MB, Drevon CA, Andersen LF. Evaluation of the odd fatty acids 15:0 and 17:0 in serum and adipose tissue as markers of intake of milk and dairy fat. Eur J Clin Nutr 2005;59(12):1417-22.

51. Smedman AE, Gustafsson IB, Berglund LG, Vessby BO. Pentadecanoic acid in serum as a marker for intake of milk fat: relations between intake of milk fat and metabolic risk factors. Am J Clin Nutr 1999;69(1): $22-9$.

52. Sun Q, Ma J, Campos H, Hu FB. Plasma and erythrocyte biomarkers of dairy fat intake and risk of ischemic heart disease. Am J Clin Nutr 2007;86(4):929-37.

53. Christakis GJ, Rinzler SH, Archer M, Hashim SA, Vanitallie TB. Effect of a serum cholesterol-lowering diet on composition of depot fat in man. Am J Clin Nutr 1965;16:243-51.

54. Shepherd J, Stewart JM, Clark JG, Carr K. Sequential changes in plasma lipoproteins and body fat composition during polyunsaturated fat feeding in man. Br J Nutr 1980;44(3):265-71.

55. Glatz JF, Soffers AE, Katan MB. Fatty acid composition of serum cholesteryl esters and erythrocyte membranes as indicators of linoleic acid intake in man. Am J Clin Nutr 1989;49(2):269-76.

56. Mata P, Garrido JA, Ordovas JM, Blazquez E, Alvarez-Sala LA, Rubio MJ, Alonso R, de Oya M. Effect of dietary monounsaturated fatty acids on plasma lipoproteins and apolipoproteins in women. Am J Clin Nutr 1992;56(1):77-83.

57. Montoya MT, Porres A, Serrano S, Fruchart JC, Mata P, Gerique JA, Castro GR. Fatty acid saturation of the diet and plasma lipid concentrations, lipoprotein particle concentrations, and cholesterol efflux capacity. Am J Clin Nutr 2002;75(3):484-91.

58. Zock PL, Katan MB. Hydrogenation alternatives: effects of trans fatty acids and stearic acid versus linoleic acid on serum lipids and lipoproteins in humans. J Lipid Res 1992;33(3):399-410.

59. Nakayama K, Bayasgalan T, Tazoe F, Yanagisawa Y, Gotoh T, Yamanaka K, Ogawa A, Munkhtulga L, Chimedregze U, Kagawa Y, et al. A single nucleotide polymorphism in the FADS1/FADS2 gene is associated with plasma lipid profiles in two genetically similar Asian ethnic groups with distinctive differences in lifestyle. Hum Genet 2010;127(6):685-90.

60. Arab L. Biomarkers of fat and fatty acid intake. J Nutr 2003;133(Suppl 3):925S-32S.

61. Hodson L, Skeaff CM, Fielding BA. Fatty acid composition of adipose tissue and blood in humans and its use as a biomarker of dietary intake. Prog Lipid Res 2008;47(5):348-80.

62. Kagawa Y, Nishijima C, Nakayama K, Iwamoto S, Tanaka A, Kamachi K, Kawabata T. Nutrigenetics of Japanese vegetarians with polymorphism in the fatty acid desaturase. J Nutr Food Sci 2016;6(3):115 .

63. Adarme-Vega TC, Lim DK, Timmins M, Vernen F, Li Y, Schenk PM. Microalgal biofactories: a promising approach towards sustainable omega-3 fatty acid production. Microb Cell Fact 2012;11:96.

64. Gillies PJ, Harris WS, Kris-Etherton PM. Omega-3 fatty acids in food and pharma: the enabling role of biotechnology. Curr Atheroscler Rep 2011;13(6):467-73.

65. Barnard ND, Scialli AR, Turner-McGrievy G, Lanou AJ, Glass J. The effects of a low-fat, plant-based dietary intervention on body weight, metabolism, and insulin sensitivity. Am J Med 2005;118(9): 991-7.

66. Fraser GE, Stram DO. Regression calibration when foods (measured with error) are the variables of interest: markedly non-Gaussian data with many zeroes. Am J Epidemiol 2012;175(4):325-31.

67. Fraser GE, Yan R. A multivariate method for measurement error correction using pairs of concentration biomarkers. Ann Epidemiol 2007;17(1):64-73.

68. Orlich MJ, Fraser GE. Vegetarian diets in the Adventist Health Study-2: a review of initial published findings. Am J Clin Nutr 2014;100(Suppl 1):353S-8S. 
69. Nijveldt RJ, van Nood E, van Hoorn DE, Boelens PG, van Norren K, van Leeuwen PA. Flavonoids: a review of probable mechanisms of action and potential applications. Am J Clin Nutr 2001;74(4):418-25.

70. Scalbert A, Johnson IT, Saltmarsh M. Polyphenols: antioxidants and beyond. Am J Clin Nutr 2005;81(1 Suppl):215S-7S.

71. Kuijsten A, Arts IC, Vree TB, Hollman PC. Pharmacokinetics of enterolignans in healthy men and women consuming a single dose of secoisolariciresinol diglucoside. J Nutr 2005;135(4):795-801.

72. Watanabe S, Yamaguchi M, Sobue T, Takahashi T, Miura T, Arai Y, Mazur W, Wahala K, Adlercreutz H. Pharmacokinetics of soybean isoflavones in plasma, urine and feces of men after ingestion of $60 \mathrm{~g}$ baked soybean powder (kinako). J Nutr 1998;128(10): $1710-5$.

73. Winnike JH, Busby MG, Watkins PB, O'Connell TM. Effects of a prolonged standardized diet on normalizing the human metabolome. Am J Clin Nutr 2009;90(6):1496-501.

74. Walsh MC, Brennan L, Malthouse JP, Roche HM, Gibney MJ. Effect of acute dietary standardization on the urinary, plasma, and salivary metabolomic profiles of healthy humans. Am J Clin Nutr 2006;84(3):531-9. 